\title{
Research on the Displacing Effect of the Internet on the Traditional Media
}

\author{
Jiang Ling and Zhang Yue
}

\begin{abstract}
By quantitative research, this paper investigates whether the displacing effect of the Internet on the traditional media exists or not through descriptive statistics and multiple regression analysis. It is concluded that there exists the time displacing effect and the Internet has taken people's time off the traditional media but only temporarily; some functions of the traditional media cannot be displaced. Thus, this paper puts forward coping strategies for the traditional media and the Internet media respectively. The traditional media should establish an operation concept that connects all media; the traditional media should seek the differentiation of function and object; the online media should give a full play to its advantage and attract more users.
\end{abstract}

Index Terms-Internet-use, preference, and old media.

\section{INTRODUCTION}

Whenever a new medium occurs, the competition between the "new" and the "old" media would awaken a series of debates. The development and wide application of the Internet has imposed significant influence on the traditional media, and the ensuing debate on the relationship between preserving the new media and abandoning the old media has never gained more attention. Until December, 2013, the number of Chinese citizens amounts to 618 million, with 53.58 million of people newly gained access to the Internet; the application rate of the Internet is $45.8 \%$, which is an increase of 3.7\% year on year. In 2013, the average time of Chinese citizens spent on the Internet reaches 25.0 hours, which is an increase of 4.5 hours year on year [1]; this trend of increase has been continuous for several years. The Internet is becoming an important part of people's daily work and life, which means that the traditional media is facing the strong competition from the Internet, the audience and increase rate of the former are declining continuously. The focus of competition between the Internet and the traditional media is the audience resource. This research starts from the change of time spent on the traditional media after the audience gain access to the Internet, and probes into the competition and cooperation strategies between the Internet and the traditional media, thus providing basis of development strategies for different media.

Manuscript received April 25, 2014; revised June 27, 2014. This work was supported in part by the financial support of the China Scholarship Council.

Jiang Ling is with the School of Literature and Journalism, Sichuan University, Chengdu, CO 610065 China (e-mail: verypp@ 163.com).

Zhang Yue is with the School of Journalism and Communication, Peking University, Beijing, CO 100871 China (e-mail: zhangyue108@gmail.com).

\section{LITERATURE REVIEW}

Audience attention is the core resource in media operation, and the distribution of attention resource reflects that competitive strength between different media. The attention resource of the audience is the source of social influence and economic performance for the media, as well as the fundamental factor for the survival of the media. Having the audience attention means the strong competiveness in the media market. The shift of audience attention provides a new perspective for researching the influence of the Internet on the traditional media, because it reflects the change of competitive strength of different media and is the comprehensive reflection of the development situation of the content and form of the media. Having an understanding of the shift of the distribution of audience attention resource and the current distribution situation provides significant reference for both the "new" and the "old" media to adjust operation strategies and participate in media competition.

Audience attention is mainly reflected in the distribution of time spent on media [2]. The time that people spent on the media each day is limited, according to the "relative invariable principle" proposed by the media scholar McCombs [3], once a new medium occurs, people have access to it through reducing the time spent on other media but not through increasing the total amount of time spent on media. Generally, the more diverse one's leisure and entertainment is, the more diverse that person's choices for new media. The appearance of a new medium will inevitably attract the attention of the audiences on already existed ones and take the time spent on them. Consequently, the time spent on the media is a perfect variable for investigating the competitive relationship between different media.

The displacing effect is a concept for describing the competition form between media, which can be divided into function displacing and time displacing. The function displacing refers to the fact that a new medium can provide better and more effective functions compared to the current media, and once the function of a medium is displaced by another medium, the former can probably be abandoned by the audiences and cease its existence in the media market. However, the real situation is that, all the functions provided by a medium for the audiences cannot be totally displaced by another new medium, thus the function displaced between media is often reflected as time displacing, namely, people are more inclined to use new media with better function so that the time spent on other media declines, thus contributing to time displacing. Compared with the current media, the new media can provide people with new and better experience on media usage. To get better media experience, the audience 
shift the time spent on the traditional media to new media. Asa consequence, one approach of investigating the competition relationship between the new and the old media is to focus on the change of time spent on the traditional media after the audiences are exposed to new media. If the time spent on the traditional media after the audiences are exposed to new media decreases, it means the new media have displacing effect on the traditional media, namely, the new media takes advantage of traditional ones in the process of competing for audience attention.

Researchers have been investigating the displacing effect between media for long but it seems that they have not formed consensus on a certain opinion. In 1958, Himmelweit and others found that, by providing leisure functions that help people escape from the real world, the TV has displacing effect on radio and leisure reading with more function and better media resources [4]. Kayany and Yelsma find out that if people spend more time on the Internet, the time for the TV reduces to a certain extent, followed by the time for the telephone and the newspaper reading; while in providing entertainment, the Internet has not replaced the TV and the newspaper [5]. Nie and Ebring, through a research based on 185 people in 84 households, conclude that the utilization of the Internet reduces their time for newspaper reading [6]; while Stempel and others believe that the utilization of the Internet can help more people to read newspapers and listen to the radio [7]. Robinson discovers that the more time people spend on the TV, the less time they spend on the radio [8]. The New Media Consortium headquartered in the USA compares the preference of the audience on using the Internet, the TV, radio, newspaper, and magazine and concludes that people prefer to obtain information on news, entertainment, sports and weather through the traditional media. Grossman finds out that the popularity of YouTube is based on the dispelling of the power of traditional mainstream media. Based on previous researches, Li Shaonan and Liang Yongzhi give a summarizing research on the displacing effect of media. They believe that the research of time displacing effect should be conducted in relative periods of time but not absolute ones, namely, the change degree of the time spent on the traditional media should be investigated after the audience gaining access to new media [9].

Of course, the relationship of displacing effect between the media is not only reflected in the pattern of "increase-decrease", it can also be the pattern of "increase-increase", namely, the utilization of one medium can promote the utilization of another one, the relationship between the TV and the video recorder is a perfect example. The video recorder can help people arrange their time to watch TV more flexibly, and this actually helps to increase the time amount of watching TV. Robinson et al conclude in their research that there exists a mutually promotive effect between online communication media and publishing media. As a consequence, the displacing effect can most probably happen between media with equal function; or those rely on the same limited resources and provide similar resource usage and satisfaction degree. [10]

Currently, there are no researches investigating the competition and cooperation relationship between the Internet and the traditional media from the perspective of media time displacing effect. This research investigates the issue from this perspective and probes into the current situation of competition between the traditional media and the Internet.

Based on what has been discussed above, this research should first discover whether the utilization of the Internet media will affect the time people spent on the traditional media. A hypothesis is put forward:

H1: The utilization of online media decreases the time people spent on the traditional media;

Furthermore, this research uses regression analysis and descriptive statistics to discover the relationship between the time spent on the Internet and on the traditional media, thus putting forward:

H2.1: The longer the time of access to the Internet, the less the time spent on the traditional media.

H2.2: The longer the time spent on the Internet each day, the less the time spent on the traditional media.

Then, this research aims to discover the relationships between the utilization of different online functions and the time spent on the traditional media. Thus 8 hypotheses are put forward:

H3.1: The more the utilization of the online game function, the less the time spent on the traditional media.

H3.2: The more the utilization of the online novel function, the less the time spent on the traditional media.

H3.3: The more the utilization of the visual pet function, the less the time spent on the traditional media.

H3.4: The more the utilization of the online friend-making function, the less the time spent on the traditional media.

H3.5: The more the utilization of the online working function, the less the time spent on the traditional media.

H3.6: The more the utilization of the online education function, the less the time spent on the traditional media.

H3.7: The more the utilization of the online shopping function, the less the time spent on the traditional media.

H3.8: The more the utilization of the online investment function, the less the time spent on the traditional media.

\section{RESEARCH METHODOLOGY}

This research uses sampling survey method; the sampling frame is all the Chinese citizens. The questionnaires are handed out from December $1^{\text {st }}, 2012$ to December $14^{\text {th }}, 2012$, and 3,000 valid questionnaires are collected, in which the number of male citizen is $1678(N=1678)$, and that of female citizen is $1313(N=1313)$. The data is analyzed using the SPSS statistic software.

By using Richter's five level scale chart, the respondents are required to self-assess the change degree of time spent on the traditional media after gaining access to the Internet.

\section{THE EFFECT OF THE INTERNET UTILIZATION ON THE Time SPENT ON THE TRAditional MEDia}

The time spent by the respondents on the newspaper, the radio, the $\mathrm{TV}$, magazines and books is integrated into a variable of "the traditional media", and by using multiple regression analysis, the correlations between time spent on 
the Internet each day, the time of access to the Internet, common online behaviors and the change of time spent on the traditional media are analyzed. The formula is shown in Table I.

TABLE I: THE MULTIPLY REGRESSION FORMULA OF COMMON ONLINE BEHAVIORS AND THE CHANGE OF TIME SPENT ON THE TRADITIONAL MEDIA (ADJUSTED $R^{2}=0.144$ )

\begin{tabular}{lll}
\hline \hline & Ratio & Distinctiveness \\
\hline Constant & 3.579 & .000 \\
Time of access to the internet & -.048 & .003 \\
Time spent on the internet each day & -.906 & .000 \\
Online games & -.012 & .441 \\
Online novel & -.013 & .417 \\
Visual pet & .058 & .000 \\
Online friend making & -.181 & .004 \\
Online working & .048 & .001 \\
Online education & -.157 & .027 \\
Online shopping & -.085 & .000 \\
Online investment & .069 & .000 \\
The square of online friend making & .053 & .000 \\
The square of online education & .037 & .002 \\
The square of time spent on the internet & .261 & .000 \\
each day & & \\
The cubic of time spent on the internet each & -.022 & .000 \\
day & & \\
\hline \hline
\end{tabular}

(By using the formula and the descriptive statistics analysis, the following conclusions are made)

\section{A. People Decrease the Time Spent on the Traditional Media Due to the Contact with the Internet}

From the formula model, it can be concluded that there exists negative correlation between the time of access to the Internet, the time spent on the Internet each day and the time spent on the traditional media, which means the more the time spent on the Internet, the less the time spent on fundamental the traditional media. By frequency statistics, more details are found. $65.8 \% \quad(N=1973)$ of the respondents indicate that the time spent on the newspaper is decreased after gaining access to the Internet; 75.4\% ( $N=2262)$ of the respondents indicate that the time spent on the radio is decreased after gaining access to the Internet, in which about half of them believe that the time has reduced to a large extent; $62 \%$ ( $N=1860$ ) of the respondents indicate that the time spent on the TV is decreased after gaining access to the Internet; $65.3 \%$ ( $N=1959$ ) of the respondents indicate that the time spent on magazines is decreased after gaining access to the Internet; $60.9 \% \quad(N=1828)$ of the respondents indicate that the time spent on books is decreased after gaining access to the Internet. Compared with the survey on Internet influence degree conducted in 2008, the ratio of the time spent on magazines has reduced, while those of other the traditional media have increased, with ratio of the time spent on the radio increasing on the biggest scale. In the survey of 2008, $68.5 \%$ of the citizens indicate that they decrease the time spent on the radio and the result of this survey shows that the ratio has increased $7.1 \%$. This trend indicates that with the wide application of the Internet, people's reliance on the Internet has increased and the time spent on the traditional media has decreased, in which the time spent on the radio decreased on the biggest scale under the influence of the

\section{Internet.}

Only $10 \%$ of the respondents indicate that their time spent on the traditional media increased after gaining access to the Internet, while more than $60 \%$ indicate that their time spent on the traditional media has decreased. Among the five the traditional media, the biggest amount of decreased time is the radio, thus, there exists the displacing effect of the Internet on the traditional media, namely, the Internet has taken people's time spent on the traditional media and $\mathrm{H} 1$ is true.

\section{B. The Time Spent on the Radio Decreased on the Biggest Scale Due to the Internet Utilization}

By comparing the average of increased and decreased time spent on the traditional media, it is discovered that the time spent on the Internet has a significant effect on the time spent on the traditional media. The time spent on the Internet is measured by the time spent on the Internet each day and the time of access to the Internet.

In terms of the time spent on the Internet each day, among the five the traditional media, it is discovered that the time spent on the radio is affected by the time spent on the Internet to the biggest extent. As is shown in Table 1, for the contact with the radio by the respondents, the two turning points are three and nine hours spent on the Internet each day. For people who spend less than three hours on the Internet each day, with the increase of the time spent on the internet, the decrease degree of the time spent on the radio becomes bigger; for people who spend three to nine hours on the Internet each day, with the increase of the time spent on the internet, the decrease degree of the time spent on the radio becomes smaller; while for people who spend more than nine hours on the Internet each day, the decrease degree of the time spent on the radio starts to become bigger. The change tendencies of the decrease degree of reading newspaper, magazine, and books correspond to that of the radio, but the former three are smaller compared with the latter. For people who spend less than five hours on the Internet each day, the decrease degree of the time spent on book is the smallest. Among those who spend less than nine hours on the Internet each day, the more the time spent on the Internet, the smaller the decrease degree of the time spent on the TV.

Measured by the time of access to the Internet (as is shown in Fig. 1), among the five the traditional media, the decrease degree of the time spent on the radio is the biggest. For those who have had access to the Internet for less than one year, the time spent on the TV has an obvious decrease; for those who have had access to the Internet for more than two year, there is no distinct difference in the decreased time spent on the TV. For those who have had access to the Internet within two years, the longer the time of access to the Internet, the bigger the decreased degree of the time spent on the newspaper. For those whose time of access to the Internet is two years, the decrease of the time spent on the newspaper is the biggest. For those whose time of access to the Internet is no more than two years, the time spent on books decreases progressively; for those whose time of access to the Internet is two to five years, the decrease degree of the time spent on books is smaller than those whose time of access to the Internet is less than two years; while for whose time of access to the Internet is more than five years, the time spent on books decreases 
greatly.

\section{Those Who Spend a Little Time on the Internet Spend More Time on the Traditional Media}

It is also concluded by Fig. 1 and Fig. 2 that besides the TV audiences, those who spend less than one hour on the Internet each day have the least decrease degree of the time spent on the traditional media. For those who spend less than three hours on the Internet each day, their time spent on the traditional media is taken to a large extent. For those who spend about three hours on the Internet each day, they spend the least time on various the traditional media. For those who spend three to nine hours on the Internet each day, the more the time spent on the Internet, the less the time taken by the traditional media. For those who spend more than nine hours on the Internet each day, the degree of preempted time by the traditional media begins to increase. As a whole, for those who have access to the Internet within two years, their time spent on the traditional media (not including the magazine) is taken by the Internet to a large extent, which means that for those who have just had access to the Internet, they shift their time on the traditional media to the Internet. With the increase of the time of access to the Internet, the decrease degree of the time spent on the traditional media maintains stable or even begin to decline; while for those whose time of access to the Internet is more than five years, the time spent on the traditional media decreases with the increase of the time of access to the Internet.

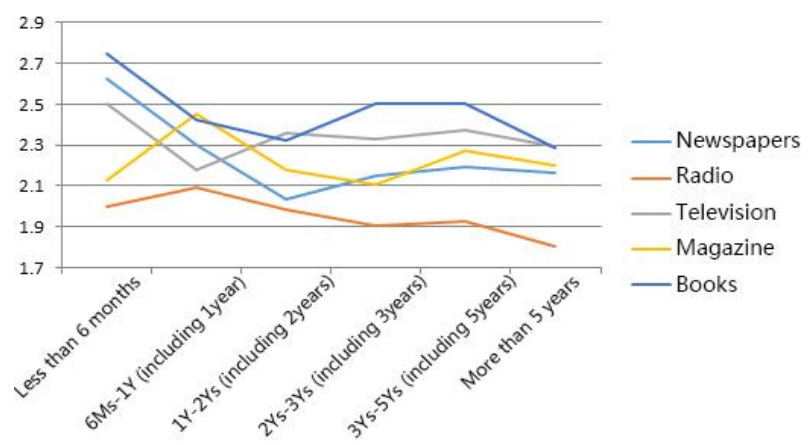

Fig. 1. The influence of the respondents' time of the access to the Internet on the time spent on the traditional media.

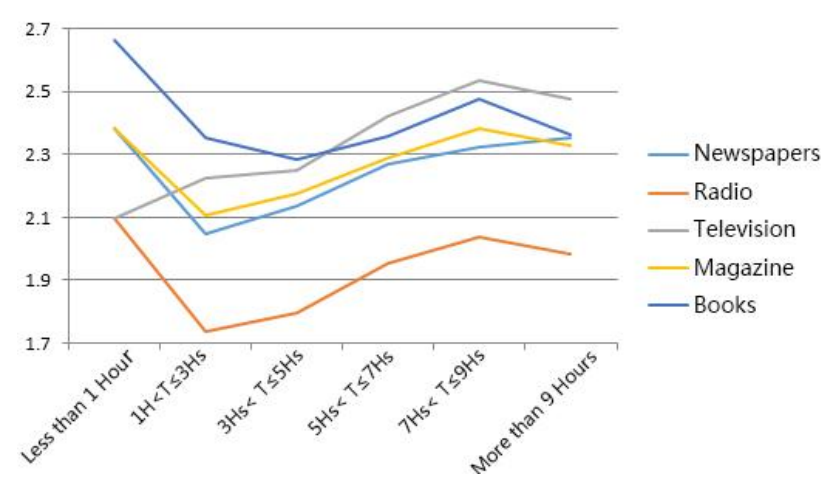

Fig. 2. The influence of the respondents' time spent on the Internet each day on the time spent on the traditional media.

On the vertical axis, the data from top to bottom represents the increase of decreased degree of the time spent on the traditional media.

\section{The Different Influence of Different Internet Usage Preference on the Time Spent on the Traditional Media}

It can be concluded from the above formula that there is no distinct relationship between the change of time spent on online game or novel and the time spent on the traditional media, while such relationship is distinct between the other six online usage behaviors and the time spent on the Internet It can be concluded in Fig. 3 that those who spend more time on online friend-making and online education, relatively spend less time on the traditional media, and those who use the online friend-making function spend less time on the traditional media compared with those who use online education function.

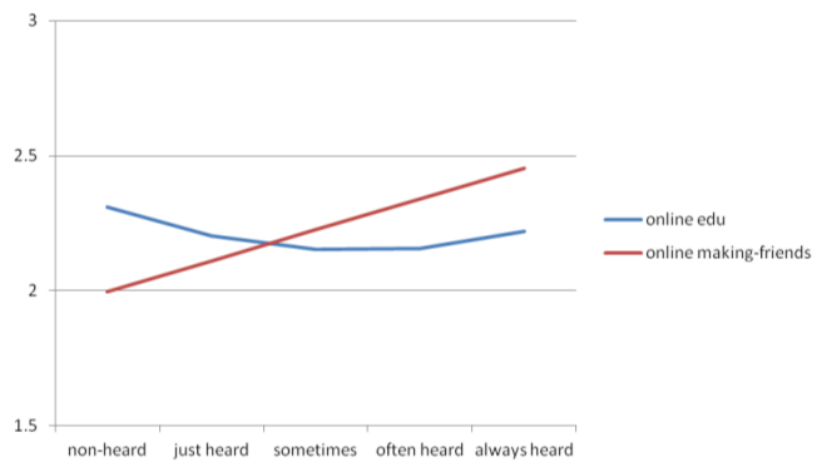

Fig. 3. The relationship between online friend-making function, online education function and the change degree of the time spent on the traditional media.

On the vertical axis, the data from top to bottom represents the increase of decreased degree of the time spent on the traditional media.

It can be concluded from Fig. 4 that those who always use the online shopping function spend the least time on the traditional media. While for those who like visual pet, online investment and online working, the more the time they spend on these online activities, the less the decreased degree of their time spent on the traditional media, namely, the increase of these online activities do not reduce the time spent on the traditional media.

Thus, hypothesis H2.1, H2.2, H3.4, H3.6 and H3.7 are proved to be valid; while hypothesis $\mathrm{H} 3.3, \mathrm{H} 3.5$ and $\mathrm{H} 3.8$ are proved to be invalid.

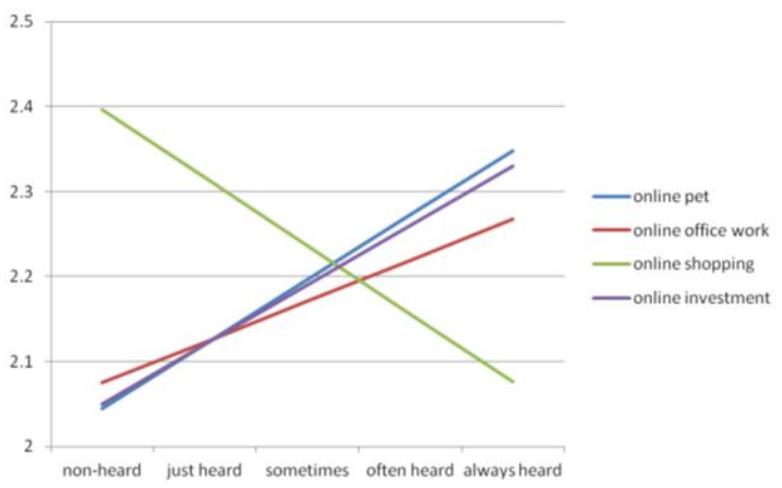

Fig. 4. The relationship between visual pet function, online working function, online shopping function, online investment function and the change degree of the time spent on the traditional media. 


\section{CONCLUSION}

From the above discussion, it can be concluded that the time people spend on the Internet has displaced that spent on the traditional media. However, some functions of the traditional media cannot be temporarily replaced by the functions of the Internet, thus maintaining certain audiences for the traditional media. To be more specific, two conclusions can be made.

\section{A. The Audience Resource of the Traditional Media is Lost}

It is concluded that there exists the time displacing effect of the Internet media on the traditional media and the time originally spent on traditional media has been shifted to the Internet and this trend has been increasing in the recent four years. More than $60 \%$ of the citizens indicate in the survey that the time they spent on the traditional media is reduced due to the usage of the Internet, in which the time spent on the radio reduces on the biggest scale while the time spent on books reduces on the smallest scale. The longer the time of access to the Internet, the less the time spent on the traditional media; and there exists distinct relationship between the time spent on the Internet each day and the time spent on the traditional media, but the increase-and-decrease relationship between the two is relatively complex.

\section{B. The Time of the Traditional Media Providing Information and Leisure Service Can Be Easily Displaced By the Internet}

The loss of audience resource of the traditional media is reflected in that the audiences' time on the traditional media is taken by the Internet, but the reason underling it is that the functions of the traditional media are displaced by the Internet. Among the specific functions provided by the traditional media such as the newspaper, the radio and the TV, the functions such as information communication, leisure, entertainment, education can easily be displaced, while other functions such as companionship, ceremony can hardly be displaced, so that the time spent on those media whose functions can hardly be displaced will not be taken by the Internet.

Reading online news, online novels and receiving online education are all activities that can help obtain information, when users are accustomed to obtaining information in a faster and more convenient manner on the Internet, they no longer resort to the information function provided by the traditional media, so their time spent on the traditional media is naturally reduced. Shopping is a leisure activity, and online shopping taken people's leisure time and displaces the leisure function provided by the traditional media, thus making the online shopper reduce the time spent on the traditional media. The activity of visual pet, online investment and online working are highly objective and task-based, and they are not the functions that can be provided to the audience by the traditional media, so that accomplishing these things will not make use of the time spent on the traditional media, thus the functions of the traditional media cannot be displaced.

\section{Suggestions}

Compared with the Internet media, some functions of the traditional have been displaced while others have not. As a consequence, the traditional media should stay attractive to their current audiences and try to retrieve the lost audiences; while the online media should give a full play to its advantage of comprehensive platform, stabilize the audience resources taken from the traditional media and compete for more audience attention.

\section{A. The Traditional Media Should Establish an Operation Concept that Connects All Media}

The Internet has become a comprehensive communication platform which integrates various media technology; the function displacing effect that cannot be realized between the traditional media can be realized in the cyberspace. It has become an indisputable fact that the Internet has taken the time of the traditional media from the audiences. Faced with the competitions brought by the Internet, the traditional media should try to find ways to combine their own features with new technology and trends. On the basis of demonstrating their own advantages, they should upgrade the networking service by combining with the Internet technology. From "the integration of the newspaper and the Internet", "the integration of the TV station and the Internet" to the integration of different media, the traditional media have been exploring the networking service for ten years and made great strides. The networking products provided by the traditional media have had their own brand influence and become strong competitor in the Internet market. The China Network Television (CNTV), developed from the China Central Television (CCTV) Website, makes use of the brand and resource of the CCTV and broadcasts video programs and many original video produced by citizens for about 1,000 hours per day, providing interactive online audio-visual services in all dimensions, and the CNTV has become the main channel in which the audience watch online TV program. In the future development, the traditional media should establish an operation concept that connects all media to a deeper extent. The production of the program content, the collecting and the editing, as well as the post-production and package should be based on the background of new media communication, and the media content and communication channel should be innovated by fully using the communication characteristics of the online platform.

\section{B. The Traditional Media Should Seek the Differentiation of Function and Object}

The internet new media is a comprehensive platform which integrates the communication advantages of all current media, and the traditional media cannot compete with the Internet with comprehensive characteristics. The traditional media should have core functions that can hardly be displaced in order to maintain current audiences by those core functions and retrieve the lost audiences taken by the Internet media. Our research has proved that there are some functions of the traditional media that cannot be displaced by the Internet, so that the best strategy of maintaining the current "territory" and retrieve the lost audiences is to develop those functions. Consequently, the traditional media should seek differentiated service in terms of object and function. The differentiation of the object is to adopt 
particular operation principle for different audience groups. This research presents the population statistics feature of the audiences lost by the traditional media and the characteristics of media contact, and clarifies the characteristics of the people who stick to the traditional media. For those remaining audiences, the differentiated communication should be conducted by analyzing the specific needs of different audience groups and discover the needs and habits of media usage of the group in terms of habit, leisure and entertainment so as to find out the breaking point for competition. For middle-aged and senior citizens, the media service should be provided continuously in their familiar and accustomed manner, especially focusing on the specificity of the content. For those lost audience groups, the basic reason that made them turn to the Internet should be discovered. The media functions of the traditional media should be developed by the online media and by integrating their own advantaged, new services should be developed with specificity, thus promoting more online users to contact with the traditional media. The differentiation of function should be based on the current contents and resources, and the current contents should be re-packaged according to the Internet communication features, for example, the multi-media database can be established and launched by the application in Mobile Terminal.

Discovering new needs of the audiences are actually a question of innovation, and the key of the innovation is to focus on the new functions that can hardly be replaced. New functions should be expanded in two aspects: the content and communication form. The innovation of the content means to expand new focus point of information and content theme; and the innovation of communication form should, on the one hand, extend the advantages of the already existed channel and develop new service forms of this medium on the other hand. The Online Spring Festival Gala is a good example of discovering new needs of the audience. It not only satisfies the family habit of watching the Spring Festival Gala together as a ceremonial event, but also integrates the form of online communication, which enriches the characteristics of interactivity and optionality of the online video communication and corresponds to the contact habit of the young people towards the media.

\section{The Online Media Should Give a Full Play to Its Advantage and Attract More Users}

Faced with the struggle and competition from the traditional media, the online media are not secure and they should try to stabilize the user resources taken from the traditional media and try to attract as many audiences as possible. As is shown by this research, as a comprehensive media platform, though the Internet has taken the time spent on the traditional media from many people, it cannot displaced all the functions of the traditional media. If the online media want to attract more users and enhance the competitiveness, they should make use of their advantages in the first place. How to integrate the advantages of the traditional media and give play to the functions of the comprehensive platform is both a chance and a challenge for the traditional media. Giving a full play to the advantage of the online platform is not simply to multiply the functions of other media, but to realize optimized utilization of the integrated media based on the communication characteristics of the Internet. To be specific, the immediacy, interactivity and the function of hypertext of the Internet should be used in media communication; and with the advantage of providing numerous recordings, videos and data, the current functions of the traditional media are comprehensively integrated, thus firmly attracting users. Technology is the biggest advantage of the online media and only by using the technological advantage to the fullest can they really displace the traditional media in terms of function and the attention resources of the audience taken from the traditional media can be firmly attracted.

It is discovered by this research that there are still $40 \%$ of the respondents who have not decreased their time spent on the traditional media due to the contact with the Internet. Those people constitute the potential audience group for the online media. For female citizens, their habit of receiving information should be developed and their needs of seeking leisure and entertainment online should be fostered. For people above the age of 40 , the Internet media should start from imitating the traditional media and establish the group's trust on the Internet and develop some application form with the function of companionship. For the citizens who have not received higher education, the online media should adjust the communication form of the content, use less words but more videos and develop the content theme attractive to their educational level.

\section{REFERENCES}

[1] CNNIC: the 33rd Statistic Report of Internet Development in China, Jan. 2014.

[2] W. Schramm, Ed., Mass Communications, 2nd ed. Urbana, IL: University of Illinois Press, 1960.

[3] M. McComb, "Mass media in the marketplace," Journalism 3 Monograph, vol. 24, pp. 1-102, 1972.

[4] H. Himmelweit, A. Oppenheim, and P. Vince, Television and the Child, Oxford University Press, London, 1958.

[5] J. Kayany and P. Yelsma, "Displacement effects of online media in the socio-economical contexts of households," Journal of Broadcasting and Electronic Media, vol. 44, pp. 215-230, 2000.

[6] N. Nie and L. Ebring. (2000). Study offers early look at how internet is changing daily life. [Online]. Available: www.stanford.edu/dept/news/ pr/

[7] F. Stempel, T. Hargrove, and J. Bernt, "Relation of growth of use of the Internet to changes in media use from 1995 to 1999," Journalism and Mass Communication Quarterly, vol. 77, pp. 71-79, 2000.

[8] J. Robinson, "Television and leisure time: a new scenario," Journal of Communication, vol. 31, pp. 120-130, winter 1981.

[9] P. S. N. Lee and L. Leung, "Assessing the displacement effects of the internet," Telematics and Informatics, vol. 25, pp. 145-155, 2008.

[10] J. P. Robinson, K. Barth, and A. Kohut, "Social impact research: Personal computers mass media and use of time," Social Science Computer Review I, vol. 5, no. 1, pp. 65-82, 1997.

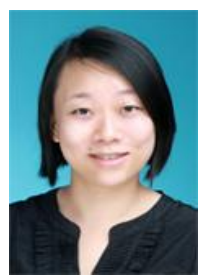

Jiang Ling was born in Xichang, Sichuan Province, China, on July 24, 1985. She is now a PhD candidate in School of Literature and Journalism, Sichuan University, majoring journalism. Before this she got her master's degree in School of Film \& TV Arts and Technology of Shanghai University. She is a visiting scholar in the School of Journalism, University of Missouri from August 2013 till now. Her research interests include new 
media and the society.

She was a journalist in Chengdu Business Daily and the Bund; a program editor in Bes TV (SMG) and International Channel of Shanghai; a teacher in the College of Media in Yunnan Normal University. She has independently published more than 10 papers in highly influential Chinese journals and 4 books, which include on chan man aesthetic value of in the context of consumerism in China film, modern literary magazine in February 2013; dissemination China 2010, Beijing: central literary contributions publishing bureau in March 2012; Footprint-ten years of Liangshan Mobile Company, Beijing: central literary contributions publishing bureau in July 2009.

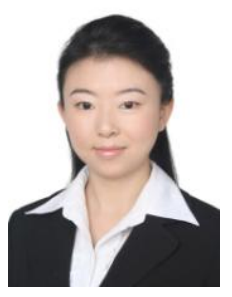

Zhang Yue was born in Chengdu, Sichuan Province, China, on July 15,1984 . She is now a PhD candidate in School of Journalism and Communication, Peking University, majoring communication. Before this, she got her bachelor's degree in Renmin University of China and master's degree in the Chinese University of Hong Kong consecutively. Her research interests include impacts of new media technologies, new media effects, public opinion online.

She has independently published 4 papers in highly influential Chinese journals, and co-wrote two books, one of which has already on shelf.

Dr. Zhang is a member of Asia media information and communication center. 